\title{
ENDOTRIVIAL MODULES FOR FINITE GROUPS OF LIE TYPE
}

\author{
JON F. CARLSON, NADIA MAZZA, AND DANIEL K. NAKANO
}

\section{INTRODUCTION}

Let $G$ be a finite group and $k$ be a field of characteristic $p>0$. An endotrivial $k G$-module is a finitely generated $k G$-module $M$ whose $k$-endomorphism ring is isomorphic to a trivial module in the stable module category. That is, $M$ is an endotrivial module provided $\operatorname{Hom}_{k}(M, M) \cong k \oplus P$ where $P$ is a projective $k G$ module. Now recall that as $k G$-modules, $\operatorname{Hom}_{k}(M, M) \cong M^{*} \otimes_{k} M$ where $M^{*}=$ $\operatorname{Hom}_{k}(M, k)$ is the $k$-dual of $M$. Hence, the functor " $\otimes_{k} M$ " induces an equivalence on the stable module category and the collection of all endotrivial modules makes up a part of the Picard group of all stable equivalences of $k G$-modules. In particular, equivalence classes of endotrivial modules modulo projective summands form a group that is an essential part of the group of stable self-equivalences.

Endotrivial modules were first defined by Dade in [Da]. He demonstrated that for $p$-groups, the endotrivial modules formed the building blocks of the endo-permutation modules which he proved are the sources for the irreducible modules in finite $p$ nilpotent groups. Later, Puig proved that in fact, the source of a simple module of a finite $p$-solvable group is an endo-permutation module. Dade also showed that if $G$ is an abelian $p$-group, then any endotrivial $k G$-module is the direct sum of a syzygy of the trivial module $\left(\Omega^{n}(k)\right.$ for some $n$, see Section 2 for a definition) and a projective module. More recently, the first author and Thévenaz have given complete classification of the endotrivial modules for $p$-groups. The group $T(G)$ of endotrivial modules is torsion-free except in the cases that the group $G$ is cyclic, quaternion or semi-dihedral [CaTh2]. The torsion-free rank of $T(G)$ was determined by Alperin $[\mathrm{A}]$. The rank depends on the number of conjugacy classes of maximal elementary abelian $p$-subgroups of $p$-rank 2 . A complete set of generators for the group of endotrivial modules was given in [CaTh3, Ca2].

The purpose of this paper is to determine the group of endotrivial modules in the defining characteristic for all finite groups of Lie type, including those of twisted type. It is well understood that if $G$ is an arbitrary finite group and $M$ is an endotrivial $k G$-module, then both the Green correspondent and the source of $M$ are endotrivial modules. For this reason we first consider the endotrivial modules for a Sylow $p$ subgroup $U$ and its normalizer $B$, a Borel subgroup, of a given finite group $G$ of Lie type. For the unipotent and Borel subgroups we present a complete classification of the endotrivial modules. For the finite groups $G$ of Lie type, $T(G)$ has rank one and is generated by the class of $\Omega(k)$ except in cases where the Lie rank is small and the

Date: July 27, 2005.

Research of the first author was supported in part by NSF grant DMS-0401431.

Research of the third author was supported in part by NSF grant DMS-0400548. 
field of the group is close to the prime field. In these exceptional cases, we find the rank of $T(G)$. It would seem that finding a complete set of generators for the group of endotrivial modules would require a more detailed knowledge of the cohomology ring $\mathrm{H}^{*}(G, k)$ than is currently available.

We end this introduction with a specific guide to the results of the paper. The definitions and preliminaries are in Section 2. In the process of classifying the endotrivial modules for finite groups of Lie type, many of the results for $p$-groups are extended to arbitrary finite groups. We first introduce the group $T(G)$ of endotrivial $k G$-modules and show that it is a finitely generated abelian group. Hence it is the direct sum of its torsion subgroup $T T(G)$ and a torsion-free subgroup $T F(G)$ which we identify with the image of the product of the restriction maps onto the groups of endotrivial modules of elementary abelian $p$-subgroups of $G$ of $p$-rank at least 2 (cf. Theorems 2.2, 2.3). In Section 3, using methods as in [Ca2], we prove that Alperin's theorem on the rank of $T(G)$ holds also for all finite groups, not just $p$-groups. Starting in Section 4, we focus on the finite groups of Lie type. In Section 5, the case where the Sylow $p$-subgroups are trivial intersection subgroups is considered. The next two sections handle the larger groups, where it turns out that $T(G)$ is cyclic. The torsion subgroup of the group of endotrivial modules is handled in Section 6, and the torsion-free rank of $T(G)$ is dealt with in Section 7. In the last section we look at the remaining cases, namely the three Chevalley groups of Lie rank 2 .

The following statements summarize the results of our investigations.

\section{Theorem A:}

(a) If $G$ is not of type $A_{1}(p)(p>2),{ }^{2} A_{2}(p)$, or ${ }^{2} B_{2}\left(2^{\frac{1}{2}}\right)$, then the torsion subgroup $T T(U)$ of $T(U)$ is trivial, by the classification of endotrival modules over $p$-groups.

(b) The torsion subgroup $T T(B)$ of $T(B)$ is isomorphic to the direct sum of $T T(U)$ and the character group of the torus $T \cong B / U$.

(c) If $G$ is not of type $A_{1}(p)(p>2),{ }^{2} A_{2}(p)$, or ${ }^{2} B_{2}\left(2^{\frac{1}{2}}\right)$, then the torsion subgroup $T T(G)$ of $T(G)$ is trivial.

The exceptional cases mentioned in Theorem A are examined in detail in Proposition 5.2. Part (b) of the theorem actually follows from general principles developed in Corollary 2.7. The torsion-free group $T F(G)$ is described as follows.

Theorem B: The ranks of $T F(U), T F(B)$ and $T F(G)$ are determined entirely by the number of conjugacy classes of maximal elementary abelian $p$-subgroups of $p$-rank 2 in the groups $U, B$ and respectively $G$.

(a) If $G$ has type $A_{1}(p)(p>2)$, or ${ }^{2} B_{2}\left(2^{\frac{1}{2}}\right)$, then $T F(U), T F(B)$ and $T F(G)$ are all trivial.

(b) If $G$ is one of the of the following, then the rank of $T F(G)$ is explored in detail in the designated statement:

(i) for type $A_{2}(p)$, cf. 8.1,

(ii) for type $B_{2}(p)$, cf. 8.2,

(iii) for type $G_{2}(p)$, cf. 8.4, 
(iv) for type ${ }^{2} A_{2}(p)$, cf. 5.4,

(v) for type ${ }^{2} B_{2}\left(2^{a+\frac{1}{2}}\right)$ (for $a \geq 1$ ), cf. Theorem 5.5,

(vi) for type ${ }^{2} G_{2}\left(3^{a+\frac{1}{2}}\right)$ (for $a \geq 0$ ), cf. Theorem 5.6.

(c) In all the other cases, the ranks of $T F(U), T F(B)$ and $T F(G)$ are one. (Theorem 7.5).

(d) A complete set of generators for $T F(U)$ and $T F(B)$ can be specified using Theorem 3.4.

It is worth stating that in the process of proving part (b) of Theorem B we enumerate the conjugacy classes of maximal elementary abelian $p$-subgroups of $G$. These conjugacy classes are in one-to-one correspondence with the components of the maximal ideal spectrum $V_{G}(k)$ of the cohomology ring $\mathrm{H}^{*}(G, k)$ (cf. Section 3). Hence the results are of some interest, independent of the structure of endotrivial modules.

\section{Definitions and Preliminaries}

Throughout the paper we assume that $k$ is an algebraically closed field of characteristic $p>0$. The algebraic closure assumption is not really necessary, but it makes the geometry easier and there is no loss in generality. For $G$ a finite group, we consider only finitely generated $k G$-modules. We begin with some general notation. Let $\bmod (k G)$ denote the category of finitely generated $k G$-modules, and let $\operatorname{stmod}(k G)$ denote the stable module category. That is, the category where the objects are finitely generated $k G$-modules. The set of morphisms between objects $M$ and $N$ in the stable category is given as

$$
\underline{\operatorname{Hom}}_{k G}(M, N)=\operatorname{Hom}_{k G}(M, N) / \operatorname{PHom}_{k G}(M, N)
$$

where $\operatorname{PHom}_{k G}(M, N)$ is the set of all homomorphisms that factor through some projective module.

Suppose that $M$ is a $k G$-module. If $\varphi: P \rightarrow M$ is a projective cover of $M$ then we let $\Omega(M)$ denote the kernel of $\varphi$. Similarly, we let $\Omega^{-1}(M)$ denote the cokernel of the injection $\vartheta: M \rightarrow Q$ where $Q$ is the injective hull of $M$. Both of these operators can be iterated so that for $n>1, \Omega^{n}(M)=\Omega\left(\Omega^{n-1}(M)\right)$ and $\Omega^{-n}(M)=\Omega^{-1}\left(\Omega^{1-n}(M)\right)$. These modules are called the syzygies of $M$, and satisfy the following properties

$$
\Omega^{m}(M) \otimes \Omega^{n}(N) \cong \Omega^{m+n}(M \otimes N) \oplus(\operatorname{proj}) \quad \text { and } \quad\left(\Omega^{n}(M)\right)^{*} \cong \Omega^{-n}\left(M^{*}\right)
$$

for all $k G$-modules $M$ and $N$ and all integers $m$ and $n$. Here, " $\otimes$ " means tensor over the base field $k$, and "( )*" indicates the $k$-dual. Also "(proj)" denotes some projective module. The first statement is a consequence of the fact that the tensor product of any module with a projective module is a projective module.

Suppose that $H$ is a subgroup of $G$. If $M$ is a $k G$-module, then its restriction to a $k H$-module is denoted $\operatorname{Res}_{G, H}(M)$. Likewise, if $N$ is a $k H$-module then the induced module is $\operatorname{Ind}_{H}^{G}(N) \cong k G \otimes_{k H} N$. When there is no chance of confusion we denote the restriction of $M$ to $H$ by the simple notation $M_{H}$.

The definition of an endotrivial module is as follows. 
Definition 2.1. A $k G$-module $M$ is endotrivial provided we have an isomorphism of $k G$-modules $\operatorname{Hom}_{k}(M, M) \cong k \oplus$ (proj).

So $M$ is an endotrivial module if its $k$-endomorphism ring is isomorphic to the trivial module in $\operatorname{stmod}(k G)$. Recall that $\operatorname{Hom}_{k}(M, M) \cong M^{*} \otimes M$. Hence, we have that if $M$ is an endotrivial module then so are $M^{*}$ and $\Omega^{n}(M)$ for all integers $n$. In particular, any $k G$-module of dimension one and any $\Omega^{n}(k)$ is an endotrivial module.

By the Krull-Schmidt Theorem it can be seen that any endotrivial module is the direct sum of an indecomposable endotrivial module and a projective module. Thus, we may define an equivalence relation on endotrivial modules, by saying that two endotrivial modules $M, N \in \bmod (k G)$ are equivalent if and only if $M$ and $N$ are isomorphic in $\operatorname{stmod}(k G)$. Let $T(G)$ denote the set of equivalence classes of endotrivial $k G$-modules, and write $[M]$ for the element in $T(G)$ that is the class of an endotrivial module $M$. Hence, if $M$ and $N$ are endotrivial, then $[M]=[N]$ if and only if there exist projective $k G$-modules $P$ and $Q$ such that $M \oplus P \cong N \oplus Q$. This is equivalent to the statement that $M^{*} \otimes N \cong k \oplus$ (proj). Furthermore, the tensor product induces an abelian group structure on $T(G)$ :

$$
[M]+[N]=[M \otimes N] .
$$

Some results of this section, such as the finite generation of $T(G)$, have been known for many years and, likely, by several people (cf. [Be1, p. 132]).

The term "endotrivial" was coined by Dade [Da], though he originally meant it only to apply to modules over $p$-groups. The classification of endotrivial modules over $p$-groups can be expressed roughly as follows [Da, A, CaTh2, CaTh3].

Theorem 2.2. Suppose that $G$ is a p-group.

(a) The group $T(G)$ is finitely generated and hence

$$
T(G)=T T(G) \oplus T F(G)
$$

where $T T(G)$ is the torsion subgroup and $T F(G)$ is a torsion-free subgroup.

(b) If $G$ is abelian then $T(G)$ is cyclic and is generated by the class $[\Omega(k)]$.

(c) $T T(G)=\{0\}$ except in the cases that $G$ is cyclic of order at least 3 , quaternion or semi-dihedral. In all cases $T T(G)$ is a finite abelian 2-group.

(d) Let $n$ be the number of conjugacy classes of maximal elementary abelian subgroups of p-rank 2 in $G$. Then, the rank of the group $T F(G)$ is

$$
\operatorname{Rank}(T F(G))=\left\{\begin{array}{cl}
n & \text { if the p-rank of } G \text { is } 2 \\
n+1 & \text { if the p-rank of } G \text { is at least } 3 \\
0 & \text { otherwise. }
\end{array}\right.
$$

If $G$ is cyclic, then $T(G)$ is cyclic of order 1 or 2 . For $G$ quaternion, $T(G) \cong$ $\mathbb{Z} / 4 \mathbb{Z} \oplus \mathbb{Z} / 2 \mathbb{Z}$ while if $G$ is semi-dihedral, $T T(G) \cong \mathbb{Z} / 2 \mathbb{Z}$ (cf. [CaTh1]). A set of generators for a torsion-free subgroup of $T(G)$ of maximal rank was constructed by Alperin in $[\mathrm{A}]$ using relative syzygies corresponding to noncentral subgroups that are contained in maximal elementary abelian $p$-subgroup of $p$-rank 2 . In [CaTh3] it was first shown that these elements generate all of $T F(G)$ and not just a subgroup 
of finite index. For the purposes of this paper, it is much more convenient to use the construction of the generators in [Ca2]. That is, the homological methods seem to be more easily generalized to non- $p$-groups. A thorough discussion of the generators of $T F(G)$ is in the next section.

Some of the following results will be more relevant to us later, for the classification in the case of the finite groups of Lie type. Note that the next proposition does not assume that $G$ is a $p$-group.

Proposition 2.3. Let $G$ be any finite group.

(a) Suppose that $E$ and $F$ are elementary abelian p-subgroups of $G$ such that both $E$ and $F$ have p-rank at least three. Let $M$ be an endotrivial $k G$-module and suppose that there exist integers $a$ and $b$ such that

$$
\operatorname{Res}_{G, E}(M) \cong \Omega^{a}\left(k_{E}\right) \oplus(\operatorname{proj}) \quad \text { and } \quad \operatorname{Res}_{G, F}(M) \cong \Omega^{b}\left(k_{F}\right) \oplus(\operatorname{proj}) .
$$

Then $a=b$ (cf. [A, Thm. 4]).

(b) A kG-module is endotrivial if and only if its restriction to every elementary abelian p-subgroup is endotrivial (cf. [CaTh1]).

(c) Let $\mathcal{E}$ denote the set of all elementary abelian p-subgroups of $G$ that have p-rank at least 2. Then the product of the restriction maps

$$
\prod_{E \in \mathcal{E}} \operatorname{Res}_{G, E}: T(G) \rightarrow \prod_{E \in \mathcal{E}} T(E)
$$

has the properties that its kernel is the torsion subgroup $T T(G)$ while its image is isomorphic to $T F(G)$.

Proof. The proofs of the first two statements follow from the given references. Only the third statement needs some explanation. We should first note that part (c) is true if $G$ is a $p$-group by the classification. In particular, the image of the map $\prod_{E \in \mathcal{E}} \operatorname{Res}_{G, E}$ is a torsion-free group. So suppose that $M$ is an indecomposable endotrivial module such that $[M]$ is in the kernel of the product of the restriction maps. Let $P$ be a Sylow $p$-subgroup of $G$. Then $M_{P} \cong L \oplus(\operatorname{proj})$ where $L$ is an indecomposable endotrivial $k P$-module. Moreover $M$ is a direct summand of $\operatorname{Ind}_{P}^{G}(L)$. The result is proved by noting that up to isomorphism, there are only finitely many indecomposable endotrivial $k P$-modules like $L$, whose restrictions to every elementary abelian subgroup of $p$-rank at least 2 are trivial. In addition, the induced module $\operatorname{Ind}_{P}^{G}(L)$, has only a finite number of direct summands. So the kernel of the product of the restrictions is a finite group.

Remark 2.4. In what follows we often identify $T F(G)$ with its image under the product of the restriction maps as in statement (c) of the previous proposition. We see in the next section that the image can be made more specific by choosing a minimal collection of elementary abelian $p$-subgroups such that the restrictions to those subgroups detect $T F(G)$.

Corollary 2.5. The group $T(G)$ is finitely generated.

Proof. By Proposition 2.3 the torsion-free subgroup $T F(G)$ of $T(G)$ is isomorphic to the image of the restriction maps to the elementary abelian $p$-subgroups of $G$ of 
$p$-rank at least 2 . There is only a finite number of such maps, and hence $T F(G)$ is finitely generated. A proof that $T T(G)$ is finite is part of the proof of part (c) of the proposition.

The reader should keep in mind that the restriction to any subgroup of an endotrivial module is an endotrivial module. Hence the restriction map induces a homomorphism on groups of endotrivial modules. Moreover, if $E$ is an elementary abelian $p$-group then any endotrivial $k E$-module has the form $\Omega^{a}(k) \oplus$ (proj) for some integer $a$.

In this paper we concentrate on the study of endotrivial modules over finite groups that are not $p$-groups. For this we need the following.

Proposition 2.6. Let $P$ be a Sylow p-subgroup of $G$, and let $H=N_{G}(P)$, the normalizer of $P$ in $G$. Then we have the following.

(a) The restriction map $\operatorname{Res}_{G, H}: T(G) \rightarrow T(H)$ is injective.

(b) If $M$ is an endotrivial $k G$-module, then $M$ is a direct summand of $\operatorname{Ind}_{P}^{G}(N)$ for some endotrivial $k P$-module $N$.

(c) Suppose that $M$ is a $k G$-module whose restriction to $P$ is an endotrivial module. Then $M$ is an endotrivial module.

(d) In the case that $G=H$ (i.e. that $P$ is normal in $G$ ), an indecomposable $k G$-module $M$ is endotrivial if and only if its restriction to $P$ is an indecomposable endotrivial module.

Proof. For part (a), we observe that if $M$ is an endotrivial module, then $M_{H} \cong N \oplus Q$ where $N$ is the Green correspondent of $M$. Also, the dimension of $M$ is relatively prime to $p$, since $p$ does not divide the dimension of $M^{*} \otimes M$. So $M$ and hence $N$ have vertex $P$. Since $M_{H}$ is an endotrivial module, $Q$ is a projective module. However, because $N$ is the Green correspondent, it is uniquely defined by $M$. So the restriction map is injective.

Part (b) is an application of the theory of vertices and sources. In particular, $M$ is a direct summand of $\operatorname{Ind}_{P}^{G}\left(M_{P}\right)$. Since $M_{P}$ is endotrivial, we are done.

For statement (c), let $U$ denote the kernel of the trace map $\operatorname{Hom}_{k}(M, M) \rightarrow k$. Then we have an exact sequence

$$
0 \rightarrow U \rightarrow \operatorname{Hom}_{k}(M, M) \rightarrow k \rightarrow 0 .
$$

We know that $M_{P}$ is an endotrivial module. Hence, on restriction to $P$, the sequence splits and $U_{P}$ is a projective module (actually, the sequence splits only because the dimension of $M$ is relatively prime to $p$ ). This implies that $U$ is a projective module, and $\operatorname{Hom}_{k}(M, M) \cong k \oplus U$. So $M$ is endotrivial.

(d) Let $M$ be an indecomposable endotrivial $k G$-module. Suppose that $M_{P} \cong$ $N_{1} \oplus N_{2} \oplus \cdots \oplus N_{t}$ where each $N_{i}$ is indecomposable, and $N_{1}$ is a source for $M$. Then $M$ is a direct summand of the induced module $\operatorname{Ind}_{P}^{G}\left(N_{1}\right)$. On the other hand, because $P$ is normal in $G$, the restriction of $\operatorname{Ind}_{P}^{G}\left(N_{1}\right)$ to $P$ is direct sum of $G$ conjugates of $N_{1}$. That is, every $N_{i}$ must be a $G$-conjugate of $N_{1}$. Now $M_{P}$ is a sum of an indecomposable endotrivial module and a projective. The only way that this can happen is if $M_{P}$ is indecomposable. This proves one half of part (d). The converse follows from statement (c). 
Corollary 2.7. Suppose that the Sylow p-subgroup $P$ of $G$ is normal in $G$ and that $G / P$ is abelian. If $P$ is not cyclic, quaternion or semi-dihedral, then $T T(G)$ is isomorphic to the character group of $G / P$.

Proof. If $M$ is an endotrivial $k G$-module such that $[M]$ is in $T T(G)$, then $M_{P}$ is isomorphic to $k \oplus$ (proj), by Theorem 2.2(c). However, this means that $[M]=[N]$ for $N$ some indecomposable summand of $\operatorname{Ind}_{P}^{G}(k)$. Because $G / P$ is abelian, $\operatorname{Ind}_{P}^{G}(k)$ is a direct sum of all of the irreducible $k G$ modules, which have dimension one. Therefore, $N$ affords a one-dimensional character of $G$.

We end this section with a characterization of $T(G)$ in a special case.

Proposition 2.8. Suppose that the Sylow p-subgroup $P$ of $G$ is a trivial intersection subgroup. Then the restriction map induces an isomorphism $T(G) \cong T\left(N_{G}(P)\right)$.

Proof. Set $H=N_{G}(P)$. Recall that $P$ is a trivial intersection subgroup of $G$ means that if $x \in G$ and $x \notin H$ then $P \cap x P x^{-1}=\{1\}$. Suppose that $N$ is an endotrivial $k H$-module. Then the Mackey formula implies that

$$
\operatorname{Res}_{G, H} \operatorname{Ind}_{H}^{G}(N) \cong \bigoplus_{H x H} \operatorname{Ind}_{H \cap x H x^{-1}}^{H} \operatorname{Res}_{H, H \cap x H x^{-1}}(x \otimes N) \cong N \oplus(\operatorname{proj}),
$$

where the sum is over a set of representatives of the $H$ - $H$ double cosets in $G$. The point is that if $x \notin H$, then $H \cap x H x^{-1}$ is a $p^{\prime}$-subgroup and any module induced from such a subgroup is projective. It follows that $\operatorname{Ind}_{H}^{G}(N)$ is an endotrivial module.

On the other hand, suppose that $M$ is an indecomposable endotrivial $k G$-module. Then its Green correspondent $N$ is an endotrivial $k H$-module and $M$ is a direct summand of $\operatorname{Ind}_{H}^{G}(N)$. Consequently, restriction and induction induce inverse homomorphisms between $T(G)$ and $T(H)$.

Remark 2.9. Proposition 2.8 holds in a more general case. Indeed, the claim still holds when the normalizer of the Sylow $p$-subgroup is a strongly $p$-embedded subgroup. However, we emphasize the statement of the result for a trivial intersection Sylow $p$-subgroup, since we need it thereafter.

Remark 2.10. Note that in general, the Green correspondent $M$ of an endotrivial $k H$-module $N$ is not an endotrivial $k G$-module. However, its restriction $M_{P}$ to $P$ is an endo-permutation module that belongs to the class of an endotrivial module (namely $N_{P}$ ).

The methods of the proof actually show that the stable categories $\operatorname{stmod}(k G)$ and $\operatorname{stmod}(k H)$ are equivalent and the equivalence is induced by the restriction and induction maps. Of course, the equivalence induces an isomorphism of groups of endotrivial modules.

\section{The TORSION-FREE RANK OF $T(G)$}

Alperin [A] determined the rank of $T F(G)$ in the case that $G$ is a finite $p$-group. Our purpose here is to show, using homological methods, that the same theorem holds for any finite group. 
Note that Proposition 2.3 proves that if the $p$-rank of $G$ is one, then $T F(G)=\{0\}$. So, in this section, we will assume that the $p$-rank of $G$ is at least 2 . The methods that we employ follow those in [Ca2]. We record the details here for the sake of completeness. In the case that the Sylow $p$-subgroup of $G$ is normal, we can also specify the generators of $T F(G)$, by a result of [Ca2].

For notation, let $\mathcal{C}$ be the collection of all maximal elementary abelian $p$-subgroups of $G$. Then we write

$$
\mathcal{C}=\mathcal{C}_{1} \cup \mathcal{C}_{2} \cup \cdots \cup \mathcal{C}_{n}
$$

where we have one of the following

(i) If the $p$-rank of $G$ is two, then $\mathcal{C}_{1}, \ldots, \mathcal{C}_{n}$ are the conjugacy classes of maximal elementary abelian $p$-subgroups of $G$, all of which are of order $p^{2}$.

(ii) If the $p$-rank of $G$ is greater than two, then the sets $\mathcal{C}_{1}, \ldots, \mathcal{C}_{n-1}$ are the conjugacy classes of maximal elementary abelian $p$-subgroups of $p$-rank two, and $\mathcal{C}_{n}$ is the collection of all maximal elementary abelian $p$-subgroup of $p$-rank larger than two.

Note that if there are no maximal elementary abelian $p$-subgroups of $p$-rank two in $G$, then $n=1$ and $\mathcal{C}=\mathcal{C}_{1}$. For each $i$, choose a maximal elementary abelian p-subgroup $E_{i} \in \mathcal{C}_{i}$.

Theorem 3.1. Assume that the p-rank of $G$ is at least 2. Then the torsion-free rank of the group $T(G)$ is the number $n$.

Proof. Recall that if $E$ is an elementary abelian $p$-subgroup of order $p^{e}$ for $e>1$ then $T(E) \cong \mathbb{Z}$, where the class of an endotrivial module $M$ corresponds to the number $a_{E}(M)=a$ such that $M_{E} \cong \Omega^{a}(k) \oplus(\operatorname{proj})$. We know that if $a_{E}(M)=0$ for all $E$, then the class of $M$ is a torsion element in $T(G)$. Moreover, if $E$ and $F$ both have $p$-rank at least three, then $a_{E}(M)=a_{F}(M)$ (cf. Proposition 2.3). Consequently, a torsion-free subgroup of $T(G)$ of maximal rank is isomorphic to the image of the map

$$
\Psi=\prod_{i=1}^{n} \operatorname{Res}_{G, E_{i}}: T(G) \rightarrow \prod_{i=1}^{n} T\left(E_{i}\right),
$$

where for each $i$, we choose $E_{i} \in \mathcal{C}_{i}$ as above. In particular, the torsion-free rank of $T(G)$ is at most $n$. If $n=1$, then there is nothing more to prove. So for the remainder of the proof assume that $n>1$.

By Quillen's Dimension Theorem (cf. [Be2, Thm. 5.1.1]), we know that

$$
V_{G}(k)=\bigcup_{E \in \mathcal{C}} \operatorname{Res}_{G, E}^{*}\left(V_{E}(k)\right),
$$

where $\operatorname{Res}_{G, E}^{*}: V_{E}(k) \longrightarrow V_{G}(k)$ is the restriction map induced on the varieties by the inclusion of $E$ in $G$. If $E$ and $F$ are conjugate then $\operatorname{Res}_{G, E}^{*}\left(V_{E}(k)\right)$ is the same as $\operatorname{Res}_{G, F}^{*}\left(V_{F}(k)\right)$. If $E \in \mathcal{C}$ has $p$-rank two, then $V_{E}(k)$ is a plane, and the intersection of $\operatorname{Res}_{G, E}^{*}\left(V_{E}(k)\right)$ with $\operatorname{Res}_{G, F}^{*}\left(V_{F}(k)\right)$, for $F$ not conjugate to $E$, is a subvariety $W$ of dimension one. In fact, the intersection $W$ is precisely the subvariety $\operatorname{Res}_{G, Z}^{*}\left(V_{Z}(k)\right)$ where $Z$ is a subgroup of order $p$ in the center of some Sylow $p$-subgroup of $G$. It follows that for some $m>0$ there exists an element $\zeta \in \mathrm{H}^{m}(G, k)$ with the property 
that $V_{G}(\zeta)$, the set of all maximal ideals containing $\zeta$, intersects $W$ transversely. It is sufficient here to choose the element $\zeta$ so that $\operatorname{Res}_{G, Z}(\zeta)$ is not nilpotent, or equivalently, so that $\operatorname{Res}_{G, Z}(\zeta) \neq 0$ and that $m$ is even if $p>2$. Assume that such an element has been chosen.

Let $\zeta^{\prime}: \Omega^{m}(k) \rightarrow k$ be a cocycle that represents $\zeta$. The kernel of $\zeta^{\prime}, L_{\zeta}$, is a module having support variety $V_{G}\left(L_{\zeta}\right)=V_{G}(\zeta)$ (cf. [Be2, Prop. 5.9.1]). Then, the support variety is disconnected. That is, we have that

$$
V_{G}\left(L_{\zeta}\right)=V_{1} \cup V_{2} \cup \cdots \cup V_{n}
$$

where

$$
V_{i}=\bigcup_{E \in \mathcal{C}_{i}} \operatorname{Res}_{G, E}^{*}\left(V_{E}\left(L_{\zeta}\right)\right)
$$

Notice here that when $i<n$ and in any case that $\mathcal{C}_{i}$ is the conjugacy class of a single maximal elementary abelian group of rank 2 , we have that $V_{i}=\operatorname{Res}_{G, E_{i}}^{*}\left(V_{E_{i}}\left(L_{\zeta}\right)\right)=$ $\operatorname{Res}_{G, E_{i}}^{*}\left(V_{E_{i}}\left(\operatorname{Res}_{G, E_{i}}(\zeta)\right)\right)$ for some element $E_{i}$ in $\mathcal{C}_{i}$. Moreover, because $V_{G}\left(L_{\zeta}\right)$ is transverse to $W$, it is necessary that $V_{i} \cap V_{j}=\{0\}$, for $i \neq j$. Consequently, $L_{\zeta}$ decomposes as

$$
L_{\zeta}=L_{1} \oplus L_{2} \oplus \cdots \oplus L_{n}
$$

where $V_{G}\left(L_{i}\right)=V_{i}($ cf. [Be2, Thm. 5.12.1]).

For any $i<n$, let $V_{i}^{\prime}=\cup_{j \neq i} V_{j}$ and let $L_{i}^{\prime}=\oplus_{j \neq i} L_{j}$. Then we have a diagram

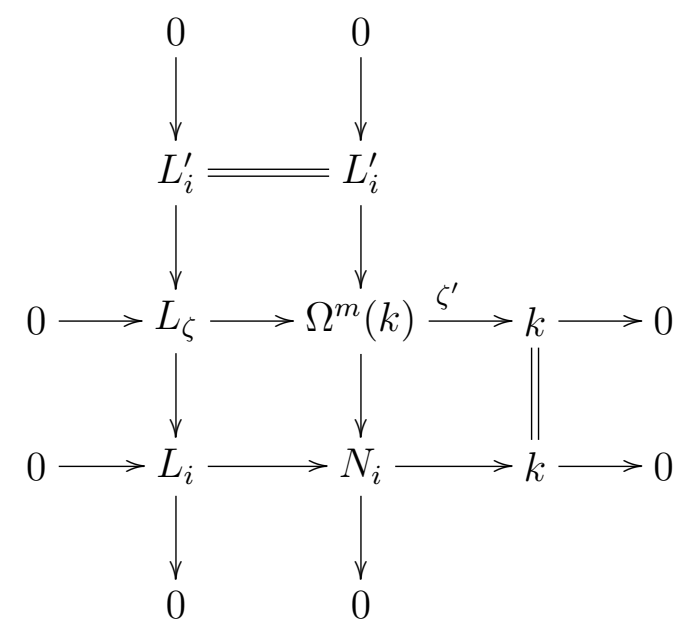

where $N_{i}$ is the pushout.

We claim that $N_{i}$ is an endotrivial module. To prove the claim it is only necessary to show that the restriction of $N_{i}$ to any maximal elementary abelian $p$-subgroup $E$ of $G$ is an endotrivial module. Suppose that $E$ is in $\mathcal{C}_{i}$. Then because $V_{i}^{\prime}$, which is the support variety of $L_{i}^{\prime}$, intersects $\operatorname{Res}_{G, E}^{*}\left(V_{E}(k)\right)$ in the set $\{0\}$ we conclude that $\operatorname{Res}_{G, E}\left(L_{i}^{\prime}\right)$ is a projective $k E$-module. Hence on restriction to the subgroup $E$, the middle column splits, and $\operatorname{Res}_{G, E} N_{i} \cong \Omega^{m}(k) \oplus$ (proj). Thus, $\operatorname{Res}_{G, E}\left(N_{i}\right)$ is an endotrivial module. On the other hand, suppose that $E \in \mathcal{C}_{j}$ for $j \neq i$. Then by a similar argument, $L_{i}$ is projective on restriction to $E$. So this time the bottom row in the diagram splits on restriction to $E$, and we have that $\operatorname{Res}_{G, E} N_{i} \cong k \oplus$ (proj). 
Hence, we have constructed a collection $N_{1}, \ldots, N_{n-1}$ of endotrivial modules. Moreover, we know the restriction of any $N_{i}$ to any maximal elementary abelian $p$-subgroup of $G$. Therefore, considering the map $\Psi$ defined at the beginning of the proof, we have that the classes of the modules $\Omega(k), N_{1}, \ldots, N_{n-1}$ generate a torsion-free subgroup of $T(G)$ that has rank $n$. This proves the theorem.

Theorem 3.1 leads us directly to the following observations.

Corollary 3.2. Let $P$ be a Sylow p-subgroup of a finite group $G$ and let $H=N_{G}(P)$.

(a) If $P$ is a dihedral group of order 8 , the torsion-free ranks of $T(G)$ and $T(H)$ equal 2.

(b) If $H$ controls the $p$-fusion in $G$, then the image of the restriction map $\operatorname{Res}_{G, H}$ is a subgroup of $T F(H)$ of finite index.

Proof. (a) In this case, $P$ has 2 maximal elementary abelian $p$-subgroups of $p$-rank 2 , and they are never conjugate inside any finite group having $P$ as a Sylow 2-subgroup.

(b) If $H$ controls the $p$-fusion, then the $H$ - and $G$-conjugacy classes of subgroups of $P$ are the same, and so $T(G)$ and $T(H)$ have the same torsion-free rank. Thus, the assertion follows, since the restriction map $\operatorname{Res}_{G, H}$ is an injective group homomorphism.

We end this section with some notes on the generators of $T F(G)$. If the group $G$ is a $p$-group, then there is a well defined formula for the generators [Ca2, CaTh3]. The generators can be constructed by carving up the syzygies of the trivial module or by taking relative syzygies as in [A]. Furthermore, the methods of [Ca2] can be extended to the case that $G$ is a finite group that has a normal Sylow $p$-subgroup.

To state the theorem we need some additional notation. Assume that $G$ has a normal Sylow $p$-subgroup $P$. We assume also that the rank of $T F(G)$ is at least two, because otherwise, we know that $T F(G)$ is generated by $\Omega(k)$. This means that the number $n$ of conjugacy classes of maximal elementary abelian $p$-subgroups of $G$ is at least 2. It follows also from the last assumption that $P$ has a cyclic center and a unique central subgroup $Z=\langle z\rangle$ of order $p$. Note that $Z$ is also normal in $G$. Let $\bar{P}=P / Z$ and $\bar{G}=G / Z$. For any $k G$-module $M$ let $\bar{M}=M / M_{0}$ where

$$
M_{0}=\left\{m \in M \mid(z-1)^{p-1} m=0\right\} .
$$

Notice that $\bar{M}$ is a $k \bar{G}$-module.

The following is a rephrasing of [Ca2, Thm. 4.2, Cor. 4.3] in the special case that $M \cong \Omega^{m}(k)$. The proof of the theorem is even more technical than its statement. It ultimately relies on a theorem of the first author and Benson on the partial inflations of cohomology of the quotient group $\bar{G}$ onto the pages of the Lyndon-HochschildSerre spectral sequence of the group extension. Cf. [Ca2] for details.

Theorem 3.3. Suppose that for some $m, V_{\bar{G}}\left(\overline{\Omega^{m}(k)}\right)=V_{1} \cup V_{2}$, where $V_{1} \cap V_{2}=\{0\}$. Assume further that for any elementary abelian p-subgroup $E$ of $G$, we have that $\operatorname{Res}_{\bar{G}}^{*}, \bar{E}\left(V_{\bar{E}}(k)\right) \subseteq V_{i}$ for either $i=1$ or for $i=2$. Then there exists an endotrivial $k G$-module $U$ with the property that one of the following happens.

(i) If $\operatorname{Res}_{\bar{G}}^{*}, \bar{E}\left(V_{\bar{E}}(k)\right) \subseteq V_{1}$, then $U_{E} \cong \Omega^{m}\left(k_{E}\right) \oplus$ (proj). 
(ii) If $\operatorname{Res}_{G, \bar{E}}^{*}\left(V_{\bar{E}}(k)\right) \subseteq V_{2}$, then $U_{E} \cong k \oplus(\operatorname{proj})$.

To state the theorem on generators we first introduce the following notation. For $i=1, \ldots, n-1$, let $a_{i}$ be defined by the following rule.

(a) If $p>2$, then let $a_{i}=2 p$.

(b) If $p=2$, then let $a_{i}=|F| / 2$ where $F$ is an elementary abelian $p$-subgroup of $\bar{P}$ which has maximal order subject to the condition that $\bar{E}_{i} \subseteq F$.

Theorem 3.4. Suppose that $G$ has a normal Sylow p-subgroup. For any $i=$ $1, \ldots n-1$ there is an endotrivial module $N_{i}$ such that

$$
\left(N_{i}\right)_{E_{i}}=\Omega^{a_{i}}(k) \oplus(\operatorname{proj}) \quad \text { and } \quad\left(N_{i}\right)_{E_{j}} \cong k \oplus(\operatorname{proj}) \quad \text { for } j \neq i .
$$

The classes $[\Omega(k)]$ and $\left[N_{i}\right]$ for $i=1, \ldots, n-1$ are a free set of generators for $T F(G)$. In particular, the restriction map induces an isomorphism $T F(G) \cong T F(P)^{G / P}$, where $T F(P)^{G / P}$ denotes the $G / P$-fixed points of $T F(P)$, for the action of $G / P$ induced by the conjugacy on the subgroups of $P$.

Proof. We know that the theorem is true in the case that $G=P$ by [Ca2, Thm. 7.2]. We fix $i$ such that $1 \leq i<n$ and let $a=a_{i}$. Recall that $\mathcal{C}_{i}$ is the $G$-conjugacy class of $E_{i}$. We write $\mathcal{C}_{i}=\mathcal{U}_{1} \cup \cdots \cup \mathcal{U}_{r}$ where each $\mathcal{U}_{j}$ is a $P$-conjugacy class of maximal elementary abelian subgroups of $p$-rank 2. The argument in the proof of $\left[\mathrm{Ca} 2\right.$, Thm. 7.2] shows that for each $j, V_{\bar{P}}\left(\overline{\Omega^{a}(k)}\right)=W_{j} \cup W_{j}^{\prime}$ where $W_{j} \cap W_{j}^{\prime}=\{0\}$, and also for any elementary abelian $p$-subgroup $E$ of $P$, $\operatorname{Res}_{\bar{P}}^{*}, \bar{E}\left(V_{\bar{E}}(k)\right) \subseteq W_{j}$ if and only if $E \in \mathcal{U}_{j}$.

The implication is that $V_{\bar{G}}\left(\overline{\Omega^{a}(k)}\right)=\operatorname{Res}_{\bar{G}}^{*}, \bar{P}\left(V_{\bar{P}}\left(\overline{\Omega^{a}(k)}\right)\right)=V_{i} \cup V_{i}^{\prime}$ where $V_{i}=$ $\cup_{j} \operatorname{Res}_{G}^{*}, \bar{P}\left(W_{j}\right)$. That is, we have that

$$
V_{\bar{P}}\left(\overline{\Omega^{a}(k)}\right)=\left(W_{1} \cup \cdots \cup W_{r}\right) \cup \hat{W}
$$

where $\hat{W}=\cap_{j} W_{j}^{\prime}$. The group $G / P$ acts on the variety $V_{\bar{P}}\left(\overline{\Omega^{a}(k)}\right)$ by permuting the components $W_{1}, \ldots, W_{r}$ since they correspond to conjugate subgroups. The subvariety $\hat{W}$ is fixed (setwise) by the action of $G / P$. The variety $V_{\bar{G}}\left(\overline{\Omega^{a}(k)}\right)$ is the orbit space of this action. Now Theorem 3.3 guarantees that there is a module $N_{i}$, which satisfies

$$
\left(N_{i}\right)_{E_{i}}=\Omega^{a_{i}}(k) \oplus(\operatorname{proj}) \quad \text { and } \quad\left(N_{i}\right)_{E_{j}} \cong k \oplus(\operatorname{proj}) \quad \text { for } j \neq i .
$$

Let $\mathcal{T}$ be the subgroup of $T F(G)$ generated by the classes $[\Omega(k)]$ and $\left[N_{1}\right], \ldots,\left[N_{n-1}\right]$. By [Ca2, Thm. 7.2], the restriction map $\operatorname{Res}_{G, P}$ takes $\mathcal{T}$ surjectively onto $T F(P)^{G / P}$. That is, the generators for $T F(P)^{G / P}$ are the orbit sums of the action of $G / P$ on $T F(P)$. On the other hand, the restriction from $T F(G)$ to $T F(P)$ is injective, by Proposition 2.3(c). It follows that the restriction map is an isomorphism on the torsion-free subgroups and that $\mathcal{T} \cong T F(G)$ as asserted.

\section{Finite groups of Lie type. Generalities}

For the remainder of the paper, we will consider the following setting. Let $\widehat{G}$ be a connected semisimple algebraic group (of adjoint type), defined over an algebraically closed field $k$ of characteristic $p>0$. Fix a maximal torus $\widehat{T}$ and let $\Phi$ be the root 
system associated to $(\widehat{G}, \widehat{T})$. The positive (resp. negative) roots are $\Phi^{+}\left(\operatorname{resp} . \Phi^{-}\right)$, and $\Delta$ is a base consisting of simple roots. Let $h$ denote the Coxeter number of the root system $\Phi$. Let $\widehat{B}$ be a Borel subgroup containing $\widehat{T}$ corresponding to the positive roots, and let $\widehat{U}$ be the unipotent radical of $\widehat{B}$. Note that $\widehat{B}=\widehat{T} \ltimes \widehat{U}$ and $\widehat{B}=N_{\widehat{G}}(\widehat{U})=N_{\widehat{G}}(\widehat{B})$. For a given root system $\Phi$ of Lie rank $n$, the simple roots will be denoted by $\alpha_{1}, \alpha_{2}, \ldots, \alpha_{n}$. We will adhere to the ordering of the simple roots as given in [BMP] (following Dynkin). For each root $\alpha \in \Phi^{+}$write $\widehat{U_{\alpha}}=\left\langle x_{\alpha}(t) \mid t \in k\right\rangle$ for the corresponding root subgroup. Then $\widehat{U}=\prod_{\alpha \in \Phi^{+}} \widehat{U_{\alpha}}$. Any $\operatorname{root} \beta \in \Phi^{+}$can be uniquely written as a linear combination $\sum_{\alpha \in \Delta} n_{\alpha} \alpha$, for non-negative integers $n_{\alpha}$. Define the height $h(\beta)$ of $\beta$ to be the positive integer $\sum_{\alpha \in \Delta} n_{\alpha}$.

Consider a Steinberg endomorphism $\sigma: \widehat{G} \longrightarrow \widehat{G}$. That is, $\sigma$ is an endomorphism of algebraic groups whose subgroup of fixed points $\widehat{G}_{\sigma}$ is finite. The finite groups $\widehat{G}_{\sigma}$ are the finite groups of Lie type, and they are defined over a finite field of characteristic $p$. They have been classified as follows (cf. [GLS, 2.2.3]). Namely, we distinguish three kinds of finite groups, according to the type of $\sigma$ :

(i) If $\sigma$ is induced by the Frobenius map on $\mathbb{F}_{q}$, for a positive power $q=p^{a}$ of $p$, then $\sigma\left(x_{\alpha}(t)\right)=x_{\alpha}\left(t^{q}\right), \forall \alpha \in \Phi^{+}$. The resulting finite groups are the Chevalley groups, or untwisted groups, usually denoted by the corresponding root system: $A_{n}(q), n \geq 1, B_{n}(q), C_{n}(q), n \geq 2$ and $D_{n}(q), n \geq 4$ are called classical, and $E_{6}(q), E_{7}(q), E_{8}(q), F_{4}(q)$ and $G_{2}(q)$ are called exceptional.

(ii) If all the roots have the same length and if $\sigma$ involves a non trivial isometry $\tau$ of order $d$ of the underlying Dynkin diagram, as well as the Frobenius map on $\mathbb{F}_{q^{d}}$, then we get the twisted groups of Lie type (or Steinberg groups). They are usually denoted by ${ }^{2} A_{n}(q),{ }^{2} D_{n}(q),{ }^{3} D_{4}(q)$ and ${ }^{2} E_{6}(q)$, where the superscript indicates the order $d$ of $\tau$. They are defined over the field $\mathbb{F}_{q^{d}}$.

(iii) Suppose that $\Phi$ is of type $B_{2}$ (resp. $F_{4}, G_{2}$ ) with $p=2$ (resp. 2, 3), and that $\sigma$ involves the Frobenius map on $\mathbb{F}_{q^{2}}$ and a non trivial permutation of the roots. These groups are defined over a finite field $\mathbb{F}_{q}$, where $q$ is an odd power of $p$. Hence, the resulting groups are the Suzuki groups ${ }^{2} B_{2}\left(2^{a+\frac{1}{2}}\right)$, and the Ree groups ${ }^{2} F_{4}\left(2^{a+\frac{1}{2}}\right)$ and ${ }^{2} G_{2}\left(3^{a+\frac{1}{2}}\right)$, defined respectively on the fields $\mathbb{F}_{2^{2 a+1}}, \mathbb{F}_{2^{2 a+1}}$ and $\mathbb{F}_{3^{2 a+1}}$, for a non negative integer $a$.

For our purposes, the reference material and the notation concerning these groups can be found in [GLS].

Let us denote by $G=\widehat{G}_{\sigma}$ one of these finite groups and the subgroups obtained from $\widehat{B}, \widehat{T}$ and $\widehat{U}$, by $B, T$ and $U$ respectively. Write also $X(T)$ for the character group of $T$.

The isometry $\tau$ induces a map from $\Phi$ to the twisted root system $\widetilde{\Phi}$ of $G$, defined as follows. Let $V$ be the Euclidian space spanned by $\Phi$ and let $\widetilde{V}=\{v \in V \mid \tau(v)=v\}$ be the set of fixed points. Then, $\widetilde{\Phi}$ consists of the projections of the roots of $\Phi$ onto $\widetilde{V}$, and the map induced by $\tau$ sends a root $\alpha$ on its projection $\widetilde{\alpha}$ in $\widetilde{V}$. 
Furthermore, we can define an equivalence relation on $\widetilde{\Phi}$ by identifying positive colinear roots. Let $\widehat{\Phi}=\{\widehat{\alpha} \mid \widetilde{\alpha} \in \widetilde{\Phi}\}$ be the set of equivalence classes, where

$$
\widehat{\alpha}=\{\beta \in \Phi \mid \exists c>0: \widetilde{\alpha}=c \widetilde{\beta}\} .
$$

So we have mappings $\Phi \rightarrow \widetilde{\Phi} \rightarrow \widehat{\Phi}$. Let $\widehat{\Delta}$ be the image of $\Delta$ under this composition of maps and $\widetilde{\Delta}$ be the image of $\Delta$ under $\Phi \rightarrow \widetilde{\Phi}$. The root subgroups of $G$ are indexed by the elements of $\widehat{\Phi}$. In particular, if $G$ is untwisted then $\Phi=\widetilde{\Phi}=\widehat{\Phi}$. In case $G$ is a Steinberg but not ${ }^{2} A_{2 m}(q)$ we have $\widetilde{\Phi}=\widehat{\Phi}$. We refer the reader to [GLS, $\S 2.3]$ for more details.

\section{THE $\operatorname{CASE}|\widehat{\Delta}|=1$}

The finite groups of Lie type for which $|\widehat{\Delta}|=1$ are $A_{1}(q),{ }^{2} A_{2}(q),{ }^{2} B_{2}\left(2^{a+\frac{1}{2}}\right)$, and ${ }^{2} G_{2}\left(3^{a+\frac{1}{2}}\right)$, according to the notation introduced above. Let us first point out a general fact concerning these groups.

Lemma 5.1. Let $G$ be such that $|\widehat{\Delta}|=1$. Then the Sylow p-subgroups of $G$ are TI subgroups.

Proof. By [GLS, Thm. 2.3.5], the Bruhat Decomposition holds in G. That is, for any $w \in N / T$ and for any $g \in G$, there exist unique $u \in U, n \in N$ and $v \in U \cap U^{w_{0} w}$ such that $g=u n v$, and the canonical projection $N \longrightarrow N / T$ maps $n$ onto $w$. Here, $N=N_{G}(T)$, and $w_{0} \in N / T$ is the unique element of $N / T$ satisfying $U \cap U^{w_{0}}=\{1\}$.

Now, if $G$ is such that $|\widehat{\Delta}|=1$, then $N / T=\left\langle w_{0}\right\rangle \cong \mathbb{Z} / 2 \mathbb{Z}$. Hence, if $g \in G$, and $g=u n v$ is its Bruhat Decomposition, then we have

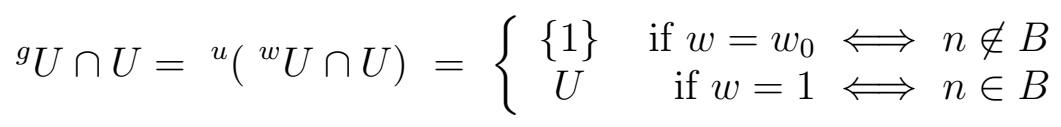

That is $U$ is a TI subgroup of $G$.

Proposition 5.2. Suppose that $G$ is isomorphic to one of $A_{1}(q),{ }^{2} A_{2}(q),{ }^{2} B_{2}\left(2^{a+\frac{1}{2}}\right)$, or ${ }^{2} G_{2}\left(3^{a+\frac{1}{2}}\right)$.

(a) If $G \cong A_{1}(2)$, then $T T(U)=T(U)=\{0\}$

(b) If $G \cong A_{1}(p)$, and $p>2$, or if $G \cong{ }^{2} B_{2}\left(2^{\frac{1}{2}}\right)$, then $T T(U)=T(U) \cong \mathbb{Z} / 2 \mathbb{Z}$.

(c) If $G \cong{ }^{2} A_{2}(2)$, then $T T(U)=T(U) \cong \mathbb{Z} / 2 \mathbb{Z} \oplus \mathbb{Z} / 4 \mathbb{Z}$.

(d) In the other cases, we have $T T(U)=\{0\}$.

Moreover, for each group we have $T T(B) \cong X(T)$, and the restriction map induces an isomorphism of $T(G)$ to $T(B)$.

Proof. (a), (b) If $G \cong A_{1}(p)$, then $U$ is cyclic of order $p$ and $\Omega^{2}(k) \cong k$, whereas $\Omega^{1}(k)$ is trivial if and only if $p=2$ (cf. [Da]). If $G \cong{ }^{2} B_{2}\left(2^{\frac{1}{2}}\right)$, then we used a computer calculation to check that the Sylow 2-subgroup is cyclic of order 4 .

(c) If $G \cong{ }^{2} A_{2}(2)$ then $U$ is a quaternion group of order 8 , and so we conclude (cf. [CaTh1, Thm. 6.3]) that $T T(U)=T(U) \cong \mathbb{Z} / 2 \mathbb{Z} \oplus \mathbb{Z} / 4 \mathbb{Z}$ (since $\mathbb{F}_{4}$ has a cubic root of unity).

(d) In all the other cases, $U$ is neither cyclic, nor quaternion, and so $T T(U)=\{0\}$, by the classification of endotrivial modules for finite $p$-groups. 
Note now that the result for $T T(B)$ follows directly from Corollary 2.7, since $U$ is a normal Sylow $p$-subgroup in $B$ and the quotient group $B / U$ is abelian. The final statement is a consequence of Proposition 2.8 and Lemma 5.1, since in all the cases we consider, we have $|\widehat{\Delta}|=1$.

In particular, if $G \cong A_{1}(q)$, then $U$ is abelian and so $T(U)$ is cyclic, generated by the class of $\Omega(k)$, and $B$ controls the $p$-fusion in $G$.

Corollary 5.3. Let $G \cong A_{1}(q)$.

(a) If $q=2$, then $U=B$, and $T(U), T(B)$, and $T(G)$ are all trivial.

(b) If $q=p>2$, then $T(G) \cong T(B) \cong T(U) \oplus X(T)$, and $T(U) \cong \mathbb{Z} / 2 \mathbb{Z}$.

(c) In all the other cases, $T(G) \cong T(B) \cong T(U) \oplus X(T)$, and $T(U) \cong \mathbb{Z}$.

Proof. Write $\Omega_{H}(k)$ for the syzygy of the trivial $k H$-module for a finite group $H$, and $\left[\Omega_{H}(k)\right]$ for its class in $T(H)$. Consider the restriction maps $\operatorname{Res}_{G, U}: T(G) \rightarrow T(U)$ and $\operatorname{Res}_{B, U}: T(B) \rightarrow T(U)$. Since $U$ is a Sylow $p$-subgroup of $G$ and $B$, we have $\operatorname{Res}_{G, U}\left(\left[\Omega_{G}(k)\right]\right)=\left[\Omega_{U}(k)\right]$, and $\operatorname{Res}_{B, U}\left(\left[\Omega_{B}(k)\right]\right)=\left[\Omega_{U}(k)\right]$. This implies that if $T(U)=\left\langle\left[\Omega_{U}(k)\right]\right\rangle$ then both restriction maps are surjective, and so the result follows from Corollary 3.2 and Proposition 5.2.

Let us now consider the other groups for which $|\widehat{\Delta}|=1$. In these cases, Proposition 5.2 answers the question regarding $T(U)$ and the torsion subgroups of the groups of endotrivial modules for $B$ and $G$.

We handle now these groups case by case and we determine the torsion-free ranks of $T(G), T(B)$ and $T(U)$. As mentioned previously, whenever $T F(G)$ is not cyclic, then our results allow us only to recover the torsion-free rank of $T(G)$, but we do not get a set of generators, except in the case where $G$ has type ${ }^{2} A_{2}(2)$, since the group is 2-nilpotent. For $T(B)$ and $T(U)$, a minimal set of generators for $T F(B)$ and $T F(U)$ can be computed using Theorem 3.4.

For brevity, for the remainder of the paper, we will write $G={ }^{d} X_{n}(q)$ instead of "a finite group $G$ of Lie type ${ }^{d} X_{n}(q)$ ", where $X \in\{A, B, C, D, E, F, G\}$ and $d=1,2$, or 3 .

$\mathbf{G}={ }^{\mathbf{2}} \mathbf{A}_{\mathbf{2}}(\mathbf{q})$ : In this case, according to [GLS, Table 2.4, p.46], the group $G$ is defined over $\mathbb{F}_{p^{2}}$ and a Sylow $p$-subgroup of ${ }^{2} A_{2}(p)$ is an extraspecial $p$-group. Namely, it is a quaternion group of order 8 , if $p=2$, and it is an extraspecial $p$-group of order $p^{3}$ and exponent $p$ otherwise.

Let us consider first the case $p>2$. We present the structure of $U$ and its normalizer $B$ in some detail, as they are found in [C1, $\S 13.7]$ (cf. pages 239-243). We may assume that the center of $U$ is generated by

$$
z=\left(\begin{array}{ccc}
1 & 0 & 0 \\
0 & 1 & 0 \\
u & 0 & 1
\end{array}\right) \quad \text { where } u \in \mathbb{F}_{p^{2}} \text { satisfies } u+u^{p}=0 .
$$

Then $z$ has order $p$.

Let $t$ be a generator of $\mathbb{F}_{p^{2}}$. Then, the set $\left\{t^{i} \mid 1 \leq i \leq p+1\right\}$ is a set of cosets representatives for $\mathbb{F}_{p}^{*}$ in $\mathbb{F}_{p^{2}}^{*}$. Moreover, there are $p+1$ elementary abelian 
$p$-subgroups of $p$-rank 2 given by $E_{i}=\left\langle x_{i}, z\right\rangle$, where

$$
x_{i}=\left(\begin{array}{ccc}
1 & 0 & 0 \\
t^{i} & 1 & 0 \\
b_{i} & t^{i p} & 1
\end{array}\right) \quad \text { with } b_{i}+b_{i}^{p}=t^{i(p+1)} .
$$

Also, the group $T \cong B / U$ is generated by

$$
\tau=\left(\begin{array}{ccc}
t^{-p} & 0 & 0 \\
0 & t^{p-1} & 0 \\
0 & 0 & t
\end{array}\right) \in T
$$

A straightforward computation gives us

$$
{ }^{\tau} x_{i}=\left(\begin{array}{ccc}
1 & 0 & 0 \\
t^{i+2 p-1} & 1 & 0 \\
t^{(p+1)} b_{i} & t^{i p+2-p} & 1
\end{array}\right) \in E_{i+2 p-1},
$$

where the index $i+2 p-1$ is taken modulo $p+1$. Therefore,

$$
\left\{{ }^{\tau^{j}} E_{1} \mid 0 \leq j \leq p-1\right\}=\left\{E_{i} \mid i=1, \ldots p-1\right\} \Longleftrightarrow 2 p-1 \not \equiv 0 \bmod (p+1) .
$$

That is, all the $E_{i}$ 's are conjugate in $B$ if and only if 3 does not divide $p+1$. If this is the case, then we have a unique conjugacy class of maximal elementary abelian $p$ subgroups of $p$-rank 2. Otherwise, we have 3 classes of such subgroups (cf. also [Hu, Satz II.10.12]). Consequently, we have the following.

Theorem 5.4. Suppose that $G={ }^{2} A_{2}(p)$ for $p \geq 3$. The torsion-free ranks of $T(U), T(B)$ and $T(G)$ are given in the table

$$
T(U) \quad T(B) \quad T(G)
$$

$$
\begin{array}{llll}
p+1 \equiv 0 \bmod 3 & p+1 & 3 & 3 \\
p+1 \neq \equiv 0 \bmod 3 & p+1 & 1 & 1
\end{array}
$$

Consider now the case $p=2$. Then, $G={ }^{2} A_{2}(2)$ is defined over $\mathbb{F}_{4}$ and it is a 2-nilpotent group of order 72 (cf. [GLS, Thm. 2.2.7], or also [Hu, Satz II.10.14]). Hence $T(G)$ and $T(B)$ are isomorphic, since their module categories $\bmod (k G)$ and $\bmod (k B)$ are equivalent. We conclude then that the restriction maps induce isomorphisms between $T(G)$ and $T(B)$ and both are isomorphic to $T(U) \oplus X(T)$, where $T(U)=T T(U) \cong \mathbb{Z} / 2 \mathbb{Z} \oplus \mathbb{Z} / 4 \mathbb{Z}$, since $\mathbb{F}_{4}$ contains a cubed root of unity (cf. Proposition 5.2).

$\mathbf{G}={ }^{\mathbf{2}} \mathbf{B}_{\mathbf{2}}\left(\mathbf{2}^{\mathbf{a}+\frac{1}{2}}\right)$ : (Suzuki groups). These groups are very specific and a computer calculation has been used to check that there are no maximal elementary abelian $p$-subgroups of $p$-rank 2. In case $a=0$, as mentioned previously, the Sylow 2subgroup is cyclic of order 4 . For larger values of $a$ the maximal elementary abelian 2-subgroups all have 2-rank at least 3. Thus we draw the following conclusion.

Theorem 5.5. Suppose that $G={ }^{2} B_{2}\left(2^{a+\frac{1}{2}}\right)$.

(a) If $a=0$, then $T(U) \cong \mathbb{Z} / 2 \mathbb{Z}$.

(b) If $a \geq 1$, then $T(U) \cong \mathbb{Z}$. 
(c) Finally, $T(G) \cong T(B) \cong T(U) \oplus X(T)$ and $T(U)$ is generated by the class of $\Omega(k)$.

$\mathbf{G}={ }^{2} \mathbf{G}_{\mathbf{2}}\left(\mathbf{3}^{\mathbf{a}+\frac{1}{2}}\right)$ : (Ree groups). As with the Suzuki groups, the Ree groups are very specific, and so a computer calculation was used to determine whether there are maximal elementary abelian 3-subgroups of order 9 in $G$.

If $a=0$, then a Sylow 3-subgroup has order 27 and is extraspecial with exponent 9 . It has a unique elementary abelian 3-subgroup of order 9 which is normal. For $a>0$, there are no maximal elementary abelian 3-subgroups of order less than 27 . Consequently we have the following theorem.

Theorem 5.6. Suppose that $G={ }^{2} G_{2}\left(3^{a+\frac{1}{2}}\right)$. Then $T(G) \cong T(B) \cong T(U) \oplus X(T)$ and $T(U) \cong \mathbb{Z}$ is generated by the class of $\Omega(k)$.

\section{The Case $|\widehat{\Delta}| \geq 2$ : Torsion subgroups}

In this section we consider the torsion groups $T T(G), T T(B)$, and $T T(U)$ for $G=\widehat{G}(q), q=p^{a}$ when $|\widehat{\Delta}| \geq 2$. First we should recall that because $U$ is a $p$-group we know $T T(U)$ from Theorem 2.2. In particular, $T T(U)=\{0\}$ except in the case that $U$ is cyclic, quaternion or semi-dihedral. This only happens when $G$ is $A_{1}(p)$ or ${ }^{2} A_{2}(2)$ (both cases having $|\widehat{\Delta}|=1$ ). Hence we have the following.

Proposition 6.1. Suppose that $|\widehat{\Delta}| \geq 2$. Then $T T(U)=\{0\}$ and $T T(B)$ is isomorphic to the character group $X(T)$ of the torus $T$.

The isomorphism is a consequence of Corollary 2.7. By Proposition 2.6, the restriction map from $T T(G)$ to $T T(B)$ is injective. Consequently, to determine $T T(G)$ we need only decide which $k B$-modules of dimension one can have a Green correspondent which is endotrivial.

Theorem 6.2. Suppose that $|\widehat{\Delta}| \geq 2$. Then $T T(G)=\{0\}$. In particular, the Green correspondent of a $k B$-module $\lambda$ of dimension one, is an endotrivial module if and only if $\lambda \cong k$.

Proof. Let $M$ be the Green correspondent of $\lambda$. It follows that $M$ is the unique indecomposable summand of $\operatorname{Ind}_{B}^{G} \lambda$ which has vertex $U$, and we can write $\operatorname{Res}_{G, B}(M)=$ $\lambda \oplus L$, for some $k B$-module $L$.

Let $n=|\widehat{\Delta}|$ and choose $\widehat{\alpha}_{i} \in \widehat{\Delta}$ with corresponding parabolic subgroup $P_{i}$. By transitivity of the restriction map, the following diagram commutes

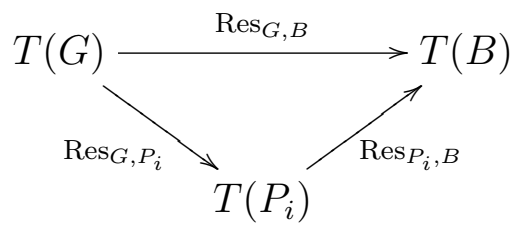

Let $N$ be an indecomposable direct summand of $\operatorname{Res}_{G, P_{i}} M$ with vertex $U$. Then $N$ is the $k P_{i}$-module which is the Green correspondent of $\lambda$. Hence, $N$ is isomorphic 
to direct summand of $M_{P_{i}}$, and $N$ is also isomorphic to a direct summand of the induced module $\operatorname{Ind}_{B}^{P_{i}} \lambda$.

The Mackey formula gives us an isomorphism of $k B$-modules

$$
\operatorname{Res}_{P_{i}, B} \operatorname{Ind}_{B}^{P_{i}} \lambda \cong \bigoplus_{B w B}\left(\operatorname{Ind}_{B^{w} \cap B}^{B} \lambda^{w}\right)=\lambda \oplus \operatorname{Ind}_{B^{w} \cap B}^{B} \lambda^{w}
$$

where the sum is over the $B$ - $B$-double cosets in $P_{i}$. The last equality follows because there are only two such double cosets.

Note that $w \neq w_{0}$ since $P_{i}$ is a proper parabolic subgroup of $G$. This forces $T$ to be a proper subgroup of $B^{w} \cap B$ (containing $T$ and the unipotent radical of $P_{i}$ ) and so we get an inequality

$$
\operatorname{Dim}\left(\operatorname{Ind}_{B^{w} \cap B}^{B} \lambda^{w}\right)=\left|B:\left(B^{w} \cap B\right)\right|<|U|
$$

Hence, the module $\operatorname{Res}_{B, U} \operatorname{Ind}_{B^{w} \cap B}^{B} \lambda^{w}$ has no projective summands. It follows that if $M$ is an endotrivial module, then $N$ also and this forces $\operatorname{Res}_{P_{i}, B} N \cong \lambda$. But the necessary and sufficient condition for

$$
\lambda=\left(\lambda_{1}, \lambda_{2}, \ldots, \lambda_{n}\right) \in X(T)
$$

to be liftable to $P_{i}$ is that $\lambda_{i}=0$ (i.e. $\lambda$ when restricted to the derived subgroup of the Levi subgroup corresponding to $P_{i}$ yields the trivial module).

It follows that if the Green correspondent $M$ of $\lambda$ is endotrivial then $\lambda_{i}=0$, for all $1 \leq i \leq n$, which is equivalent to saying that $\lambda=k$ and hence $M=k$.

\section{The CASE $|\widehat{\Delta}| \geq 2$ : TORSION-FREE RANKS}

The goal of this subsection is to show that, most of the time, the group of endotrivial modules $T(G)$ is cyclic, generated by the class of $\Omega(k)$. This will reduce the problem of determining $T(G)$ for group of Lie type to a few special cases which are treated in the next section.

Assume throughout the section that $G=\widehat{G}(q)$ for $q=p^{a}$ defined over $\mathbb{F}_{q^{d}}$, where $d$ is the order of the Steinberg automorphism acting on $\widehat{G}$. Assume also that for $G$ we have $|\widehat{\Delta}| \geq 2$.

Theorem 7.1. Suppose that $|\widehat{\Delta}| \geq 2$ and $q=p^{a}$ where $a \geq 2$. Then

(a) $T(U)=T F(U)=\langle[\Omega(k)]\rangle \cong \mathbb{Z}$,

(b) $T(B) \cong T F(B) \oplus X(T)$, where $T F(B)=\langle[\Omega(k)]\rangle \cong \mathbb{Z}$.

(c) $T(G)=T F(G)=\langle[\Omega(k)]\rangle \cong \mathbb{Z}$.

Proof. The center of $U$ has $p$-rank at least $a$. Hence if $a \geq 2$ then $U$ can not have a maximal elementary abelian subgroup of $p$-rank 2 . Consequently $T(U) \cong \mathbb{Z}$ by Theorem 2.2. The remainder follows from Corollary 2.7 and Theorem 6.2.

We now consider the case when $q=p$ (i.e. $a=1$ ) with $|\widehat{\Delta}| \geq 2$. Our analysis of this case starts by investigating the elementary abelian subgroups in the Sylow $p$-subgroups of the untwisted groups and the Steinberg groups which are not isomorphic to ${ }^{2} A_{2 m}(p)$. That is, we consider all the situations when $\widetilde{\Phi}=\widehat{\Phi}$. Then, we can prove the following. 
Lemma 7.2. Suppose that $|\widetilde{\Delta}| \geq 3$. Then there exist roots $\beta_{1}, \ldots, \beta_{s} \in \widetilde{\Phi}^{+}$, for some integer $s \geq 3$, that satisfy the following properties:

(a) There exists an integer $t$ with $1<t<s$ and $\beta_{1}, \ldots, \beta_{t}$ have the same height $h\left(\beta_{i}\right)=d>1$, for every $1 \leq i \leq t$.

(b) If $\beta \in \widetilde{\Phi}$ and $h(\beta)>d$, then $\beta=\beta_{j}$ for some $t+1 \leq j \leq s$.

(c) $\beta_{i}+\beta_{j} \notin \widetilde{\Phi}$, for every $1 \leq i, j \leq s$.

Proof. The proof is by a case by case analysis using the tables of positive roots in $[\mathrm{BMP}]$. We let the set of simple roots $\Delta=\left\{\alpha_{1}, \ldots, \alpha_{n}\right\}$ is ordered according the Dynkin ordering (as in [BMP]). In the list below we display the first $t$ roots in the list $\beta_{1}, \ldots, \beta_{s}$. The displayed roots all have height $d$. The remainder of the list consists of the roots of height greater than $d$.

(i) Let $\widetilde{\Phi}=A_{n}, n \geq 3$. Then $s=3, t=2$ and $d=n-1$ with

$$
\beta_{1}=\sum_{i=2}^{n} \alpha_{i} \text { and } \beta_{2}=\sum_{i=1}^{n-1} \alpha_{i}
$$

(ii) Let $\widetilde{\Phi}=B_{n}, n \geq 3$. Then $s=4, t=2$ and $d=2 n-3$ with

$$
\beta_{1}=\alpha_{2}+2 \sum_{i=3}^{n} \alpha_{i} \text { and } \beta_{2}=\sum_{i=1}^{3} \alpha_{i}+2 \sum_{i=4}^{n} \alpha_{i} .
$$

(iii) Let $\widetilde{\Phi}=C_{n}, n \geq 3$. Then $s=4, t=2$ and $d=2 n-3$ with

$$
\beta_{1}=2 \sum_{i=2}^{n-1} \alpha_{i}+\alpha_{n} \text { and } \beta_{2}=\alpha_{1}+\alpha_{2}+2 \sum_{i=3}^{n-1} \alpha_{i}+\alpha_{n} .
$$

(iv) Let $\widetilde{\Phi}=D_{4}$. Then $s=5, t=3$ and $d=3$ with

$$
\beta_{1}=\sum_{i=2}^{4} \alpha_{i}, \beta_{2}=\alpha_{1}+\alpha_{2}+\alpha_{4} \text { and } \beta_{3}=\sum_{i=1}^{3} \alpha_{i} \text {. }
$$

(v) Let $\widetilde{\Phi}=D_{n}, n \geq 5$. Then $s=4, t=2$ and $d=2 n-5$ with

$$
\beta_{1}=\alpha_{2}+2 \sum_{i=3}^{n-3} \alpha_{i}+\alpha_{n-1}+\alpha_{n} \text { and } \beta_{2}=\sum_{i=1}^{3} \alpha_{i}+2 \sum_{i=4}^{n-3} \alpha_{i}+\alpha_{n-1}+\alpha_{n} .
$$

(vi) Let $\widetilde{\Phi}=E_{6}$. Then $s=5, t=2$ and $d=8$ with

$$
\beta_{1}=\alpha_{1}+\alpha_{2}+2 \alpha_{3}+2 \alpha_{4}+\alpha_{5}+\alpha_{6} \text { and } \beta_{2}=\alpha_{1}+2 \alpha_{2}+2 \alpha_{3}+\alpha_{4}+\alpha_{5}+\alpha_{6} \text {. }
$$

(vii) Let $\widetilde{\Phi}=E_{7}$. Then $s=6, t=2$ and $d=13$ with $\beta_{1}=\alpha_{1}+2 \alpha_{2}+3 \alpha_{3}+3 \alpha_{4}+2 \alpha_{5}+\alpha_{6}+\alpha_{7}$ and $\beta_{2}=\alpha_{1}+2 \alpha_{2}+3 \alpha_{3}+2 \alpha_{4}+2 \alpha_{5}+\alpha_{6}+2 \alpha_{7}$.

(viii) Let $\widetilde{\Phi}=E_{8}$. Then $s=8, t=2$ and $d=23$ with

$$
\begin{gathered}
\beta_{1}=\alpha_{1}+2 \alpha_{2}+3 \alpha_{3}+4 \alpha_{4}+5 \alpha_{5}+4 \alpha_{6}+2 \alpha_{7}+2 \alpha_{8} \text { and } \\
\beta_{2}=\alpha_{1}+2 \alpha_{2}+3 \alpha_{3}+4 \alpha_{4}+5 \alpha_{5}+3 \alpha_{6}+2 \alpha_{7}+3 \alpha_{8} .
\end{gathered}
$$


(ix) Let $\widetilde{\Phi}=F_{4}$. Then $s=6, t=2$ and $d=7$ with

$$
\beta_{1}=\alpha_{1}+2 \alpha_{2}+2 \alpha_{3}+2 \alpha_{4} \text { and } \beta_{2}=\alpha_{1}+2 \alpha_{2}+3 \alpha_{3}+\alpha_{4} .
$$

The root subgroups $U_{\widehat{\beta}}, \widehat{\beta} \in \widehat{\Phi}$ in the untwisted groups and the Steinberg groups $G(q)$ which are not ${ }^{2} A_{2 m}(q)$ are isomorphic to either $\mathbb{F}_{q}$ or $\mathbb{F}_{q^{2}}$ (cf, [GLS, Table $2.4])$. In either case the root subgroups are elementary abelian $p$-groups. Let $m_{\beta}$ be the $p$-rank of $U_{\widehat{\beta}}$. Assume that we have the same hypothesis and notation as in the above proposition. Then we can prove the following.

Proposition 7.3. Let $G$ be an untwisted group or a Steinberg group which is not ${ }^{2} A_{2 m}(q)$. Let $H$ be the subgroup of $U$ generated by the subgroups $U_{\widehat{\beta}_{i}}$ for $1 \leq i \leq s$ and let $u \in U$. Set $m(j)=\sum_{i=1}^{j} m_{\beta_{i}}$ for $j=s, t$. Then

(a) $H$ is an elementary abelian p-subgroup of order $p^{m(s)}$ and $H$ is normal in $U$,

(b) $\left|C_{H}(u)\right| \geq p^{m(t)}$, and

(c) $C_{U}(u)$ has an elementary abelian $p$-subgroup of p-rank at least $m(t)+1$.

Proof. Let us recall that our assumption on $G$ implies that $\widetilde{\Phi}=\widehat{\Phi}$, and so $\widehat{\Phi}$ is also a root system.

For (a), let $x_{i} \in U_{\widehat{\beta}_{i}}$ for $1 \leq i \leq s$. Then, for any $1 \leq i, j \leq s$ we have that $x_{i}^{p}=1$ and $\left[x_{i}, x_{j}\right]=1$, since there are no roots in $\widehat{\Phi}$ of the form $a \widehat{\beta}_{i}+b \widehat{\beta}_{j}$ for any integers $a, b \geq 1$. So $H$ is elementary abelian of order $p^{m(s)}$.

Suppose that $\widehat{\gamma} \in \widehat{\Phi}$ is any positive root and consider the commutator of the elements $x_{\widehat{\beta_{i}}}(c) \in U_{\widehat{\beta}_{i}}$ and $x_{\widehat{\gamma}}(d) \in U_{\widehat{\gamma}}$, for constants $c$ and $d$ in the appropriate field. The Chevalley Commutator Formula [GLS, Thm. 2.4.5] gives us

$$
\left[x_{\widehat{\beta}_{i}}(c), x_{\widehat{\gamma}}(d)\right]=\prod_{a, b>0} x_{a \widehat{\beta}_{i}+b \widehat{\gamma}}\left(c_{a b}\right)
$$

where the product is over all positive $a$ and $b$ such that $a \widehat{\beta}_{i}+b \widehat{\gamma} \in \widehat{\Phi}$, and where the coefficients $c_{a b}$ depend on $a, b, c, \widehat{\beta}_{i}$ and $\widehat{\gamma}$. But then, $a \widehat{\beta}_{i}+b \widehat{\gamma}=\widehat{\beta}_{j}$ for some $1 \leq j \leq s$ because the height of $a \widehat{\beta}_{i}+b \widehat{\gamma}$ is greater than $d$. So $\left[x_{\widehat{\beta}_{i}}(c), x_{\widehat{\gamma}}(d)\right] \in H$, Hence, $H$ is normal in $U$.

For (b), let $J$ be the subgroup generated by all the subgroups $U_{\widehat{\beta}_{i}}$, with $t+1 \leq i \leq$ $s$. Then $J$ has order $p^{m(s)-m(t)}$. From the proof of (b), we deduce that $\left[x_{\widehat{\beta}_{i}}(c), u\right] \in J$ for any $x_{\widehat{\beta}_{i}}(c) \in H$, and for any $u \in U$. Thus for any $x_{i} \in H, x_{i} u x_{i}^{-1}=u w$, for some $w \in J$. So the $H$-conjugacy class $H \cdot u$ contains at most $|J|=p^{m(s)-m(t)}$ elements. Hence, the Class Equation gives

$$
\left|C_{H}(u)\right|=\frac{|H|}{|H \cdot u|} \geq \frac{p^{m(s)}}{p^{m(s)-m(t)}}=p^{m(t)} .
$$

If $u \in H$ then $H \subseteq C_{U}(u)$, since $H$ is elementary abelian. Now, $|H|=p^{m(s)} \geq$ $p^{m(t)+1}$, and so the part (c) holds in this case. On the other hand, suppose that 
$u \notin H$. Then

$$
C_{H}(u) \times\langle u\rangle \leq C_{U}(u) \text { and }\left|C_{H}(u) \times\langle u\rangle\right|=\left|C_{H}(u)\right| \cdot|\langle u\rangle| \geq p^{m(t)+1}
$$

by part (b).

Consider now the case $G={ }^{2} A_{2 m}(p)$ where $m \geq 2$. If $m=1$ then $|\widehat{\Delta}|=1$. The next result proves that when $m \geq 2$ the conclusion of previous proposition applies.

Proposition 7.4. Let $G={ }^{2} A_{2 m}(p)$ where $m \geq 2$. Then for any $u \in U$ of order $p$, its centralizer $C_{U}(u)$ in $U$ contains an elementary abelian subgroup of p-rank at least 3 .

Proof. By the results in [GLS, $\S 2.4]$, we know that $\widehat{\Phi}$ is the union of root systems $\Phi_{\widehat{\alpha}}$ of type $A_{1} \times A_{1}$ or $A_{2}$. More precisely, we have

$$
\Phi_{\widehat{\alpha}}=\left\{\begin{array}{cc}
\{\alpha, \tau(\alpha)\} & \text { if } \alpha+\tau(\alpha) \notin \Phi \\
\{\alpha, \tau(\alpha), \alpha+\tau(\alpha)\} & \text { if } \alpha+\tau(\alpha) \in \Phi
\end{array}\right.
$$

In the first case, $\Phi_{\widehat{\alpha}}$ is of type $A_{1} \times A_{1}$ and in the second case, $\Phi_{\widehat{\alpha}}$ is of type $A_{2}$.

Set $\beta_{i, j}=\sum_{l=i}^{i+j-1} \alpha_{l}, 1 \leq j \leq 2 m+1-i$, for $1 \leq i \leq 2 m$. Hence,

$$
\Phi^{+}=\left\{\beta_{i, j} \mid 1 \leq j \leq 2 m+1-i, 1 \leq i \leq 2 m\right\},
$$

and also $h\left(\beta_{i, j}\right)=j$, for all $i, j$. Let $\tau$ denote the symmetry (of order 2) acting on the Dynkin Diagram. Since $\tau\left(\beta_{i, j}\right)=\beta_{2 m+2-i-j, j}$, exactly one of $i$ or $2 m+2-i-j$ is less than $m$ for any given $i$. Thus, we may take

$$
\widehat{\Phi}^{+}=\left\{\Phi_{\widehat{\beta_{i, j}}} \mid 1 \leq j \leq 2 m+1-i, 1 \leq i \leq m\right\},
$$

Notice that $\Phi_{\widehat{\beta_{i, j}}}$ has type $A_{2}$ if and only if $\beta_{i, j}+\tau\left(\beta_{i, j}\right) \in \Phi$, that is, if and only if

$$
\beta_{i, j}+\beta_{2 m+2-i-j, j}=\sum_{l=i}^{i+j-1} \alpha_{l}+\sum_{l=2 m+2-i-j}^{2 m+1-i} \alpha_{l} \in \Phi .
$$

But this is a root precisely when $i+j=2 m+2-i-j$, by the structure of roots in $A_{n}$. By the definition of the $\beta_{i, j}$ 's, this corresponds to the case when the last index of the $\alpha_{l}$ 's appearing in the decomposition of $\beta_{i, j}$ is equal to $m$.

Keeping this in mind, let us now consider the following elements of $U$ :

$$
x_{j}(t)=x_{\beta_{1, j}}(t) x_{\beta_{2 m+1-j, j}}\left(t^{p}\right)
$$

for $t \in \mathbb{F}_{p^{2}}, m+1 \leq j \leq 2 m-1$ and

$$
x_{2 m}(t)=x_{\beta_{1,2 m}}(t),
$$

for $t \in \mathbb{F}_{p}$. Let $H$ be the subgroup of $U$ generated by all these elements $x_{j}(t)$, and let $J$ be the subgroup of $H$ generated by all the $x_{j}(t)$ with $j \geq m+2$. Then, by the Chevalley Commutator Formula, and [GLS, Thm. 2.4.5], the groups $H$ and $J$ are elementary abelian, of order $p \cdot\left(p^{2}\right)^{m-1}$ and $p \cdot\left(p^{2}\right)^{m-2}$, respectively. Moreover, by the Chevalley Commutator Formula we deduce as in the proof of Proposition 7.3 that $H$ is a normal subgroup of $U$, and that the $H$-conjugacy class of any element of order $p$ of $U$ lies in $J$. Hence the conclusion of Proposition 7.3 still holds for $G={ }^{2} A_{2 m}(p), m \geq 2$, since for $m \geq 2$ the index $|H: J|$ is at least $p^{2}$. 
The above propositions lead directly to the following theorem.

Theorem 7.5. Let $G=\widehat{G}\left(p^{a}\right)$ be a finite group of Lie type, (as introduced at the beginning of Section 4) with $|\widehat{\Delta}| \geq 2$. Assume that $G$ is not one of the following groups

(i) $A_{2}(p)$,

(ii) $B_{2}(p)$,

(iii) $G_{2}(p)$.

Then any maximal elementary abelian p-subgroup of $U$ has p-rank at least 3 . Moreover, we have

(a) $T(U) \cong \mathbb{Z}$.

(b) $T(B) \cong \mathbb{Z} \oplus X(T)$.

(c) $T(G) \cong \mathbb{Z}$.

In each statement, the class of $\Omega(k)$ generates a torsion-free subgroup of the group of endotrivial modules.

Proof. By Theorem 7.1, we need only consider the case when $q=p$. So, let us consider $G=G(p)$. First, suppose that $G$ is an untwisted group or a Steinberg group which is not ${ }^{2} A_{2 m}(p)$. Let $E$ be an elementary abelian $p$-subgroup of $U$ of order $p^{2}$ and consider its intersection with the unique cyclic subgroup $Z$ of order $p$ in the center of $U$. Then $E \cap Z$ has order 1 or $p$. In the first case, then $E Z$ is elementary abelian of $p$-rank 3 and contains (strictly) $E$. In the second case, let $u$ be a non central element of $E$, and consider its centralizer $C_{U}(u)$. By Proposition 7.3, $C_{U}(u)$ contains an elementary abelian $p$-subgroup $H$ of order at least $p^{m(t)+1}$. Now, since $H$ and $E=\langle u\rangle \times Z$ are subgroups of $C_{U}(u)$, and $Z$ is central, we have that $E H$ is elementary abelian of $p$-rank at least $m(t)+1$ and it contains $E$. In the case of the untwisted groups when $q=p$, we have $m(t)+1 \geq 3$ as long as $|\Delta| \geq 3$, by Lemma 7.2. Hence there are no maximal elementary abelian $p$-subgroups of $p$-rank 2 in $U$, and the result follows in the untwisted case.

Next assume that $G$ is a Steinberg group which is not ${ }^{2} A_{2 m}(p)$. Since $\widetilde{\Phi}=\widehat{\Phi}$, we can use the same reasoning as above with the root systems given in [GLS, Prop. 2.3.2] to deduce that all maximal elementary subgroups of $U$ have $p$-rank at least 3 in all cases except possibly ${ }^{2} A_{3}(p)\left(\widehat{\Phi}\right.$ has type $\left.C_{2}\right)$ and ${ }^{3} D_{4}(p)\left(\widehat{\Phi}=G_{2}\right)$. In the case when $G={ }^{2} A_{3}(p)$ consider the roots $\beta_{1}=\alpha_{1}+\alpha_{2}$ and $\beta_{2}=2 \alpha_{1}+\alpha_{2}$. These roots satisfy the properties of Lemma 7.2 for $t=1$ and $s=2$. One can now apply Proposition 7.3 with $H$ generated by $U_{\beta_{1}}$ and $U_{\beta_{2}}$ to conclude that $u$ is contained in an elementary abelian $p$-subgroup of $p$-rank at least $m(t)+1=m_{\beta_{1}}+1=2+1=3$. A similar argument holds for $G={ }^{3} D_{4}(p)$. Now assume that $G$ is ${ }^{2} A_{2 m}(p)$. For $m>1$, the results follows by Proposition 7.4.

Finally consider the Ree group ${ }^{2} F_{4}\left(2^{\frac{1}{2}}\right)$. A computer run using MAGMA (cf. [BoCan]) checks that a Sylow 2-subgroup has order $2^{12}=4096$ and it has two maximal elementary abelian 2-subgroups both having order $2^{5}=32$.

The second part of the theorem is a direct consequence Proposition 6.1, and Theorem 6.2. 


\section{TORSION-FREE RANKS FOR UNTWISTED GROUPS}

At this point we are left with computing the groups of endotrivial modules for the finite groups $G=\widehat{G}(q)$ of Lie type that are excluded from Theorem 7.5. That is when $G$ is one of $A_{2}(q), B_{2}(q)$, or $G_{2}(q)$. Notice also that Proposition 6.1 and Theorem 6.2 handle the torsion subgroups of the groups of endotrivial modules $T T(U), T T(B)$, and $T T(G)$. Hence, now, we are only concerned with the torsion-free groups $T F(G), T F(B)$, and $T F(U)$. Moreover, Theorem 7.1 reduces the question to the case $q=p$.

Recall that the generators of $T F(U)$ and $T F(B)$ can be computed from Theorem 3.4. Our investigations enable us to exhibit only the torsion-free rank of $T F(G)$.

As before, for elements in the finite groups $G$ we follow the notation of [GLS] (which is the same as in [St]). For brevity, we write $x_{\alpha}=x_{\alpha}(1)$.

$\mathbf{G}=\mathbf{A}_{\mathbf{2}}(\mathbf{p})$ : In this case, $G$ is isomorphic to the group PSL(3,p) (cf. [GLS, §2.7]). The Sylow $p$-subgroup $U$ of $G$ is an extraspecial $p$-group of order $p^{3}$ and exponent $p$, if $p>2$, and $U$ is a dihedral group of order 8 , in case that $p=2$.

If $p=2$, then $U$ has two maximal elementary abelian 2-subgroups which are not conjugate in $U$ or in $G$. Consequently, the groups $T F(U), T F(B)$ and $T F(G)$ have 2-rank 2 (cf. Corollary 3.2).

If $p>2$, then all the elements of $U$ have order $p$ and there are $p+1$ maximal elementary abelian $p$-subgroups which are normal in $U$. Suppose that $\alpha$ and $\beta$ are simple roots so that $U$ is generated by $x_{\alpha}$, and $x_{\beta}$. Then the elementary abelian $p$-subgroups in $U$ consist of the subgroups $E_{0}=\left\langle x_{\alpha}, x_{\alpha+\beta}\right\rangle$ and $E_{p}=\left\langle x_{\beta}, x_{\alpha+\beta}\right\rangle$, and the subgroups $E_{i}=\left\langle x_{\alpha} x_{\beta}^{i}, x_{\alpha+\beta}\right\rangle$ for $i=1, \ldots, p-1$. It is easy to check that the subgroups $E_{0}, E_{p}$, and $E_{1}$ are in distinct $G$-conjugacy classes. On the other hand, the set $\left\{E_{i} \mid i=1, \ldots, p-1\right\}$ is a single $B$-conjugacy class. Consequently, we have the following.

Theorem 8.1. Suppose that $G=A_{2}(p)$. The torsion-free ranks of $T(U), T(B)$ and $T(G)$ are given in the table.

$\begin{array}{cccc} & T(U) & T(B) & T(G) \\ & & & \\ p=2 & 2 & 2 & 2 \\ p 3 & p+1 & 3 & 3\end{array}$

$\mathbf{G}=\mathbf{B}_{\mathbf{2}}(\mathbf{p})$ : Let $\alpha$ and $\beta$ denote the roots with $\alpha$ a short root. The cases in which $p=2$ or $p=3$ can be handled by computer calculations. The computer algebra system MAGMA (cf. [BoCan]) was used to check our answers.

In case $p=2$, we know by [Hu, Hauptsatz II.9.22] that $G \cong \Sigma_{6}$, the symmetric group on 6 letters. The Sylow 2-subgroup $U$ has order 16 and its center is elementary abelian of order 4 . It can be checked directly from the standard relations (cf. [St], page 30) that both $x_{\alpha}$ and $x_{\alpha+\beta}$ are in the center. Hence, there are no maximal elementary abelian 2-subgroups of 2-rank 2 .

If $p=3$, then $U$ has exponent 9, since any regular element (i.e. any element of the form $x_{\alpha}^{a} x_{\beta}^{b} x_{\alpha+\beta}^{c} x_{2 \alpha+\beta}^{d}$ with $a$ and $b$ both not multiples of 3 ) has order 9 . This is 
a consequence of the calculation of the unipotent variety in [CLNP]. Consequently, every element of order 3 is contained in one of the subgroups

$$
U_{1}=\left\langle x_{\alpha}, x_{\alpha+\beta}, x_{2 \alpha+\beta}\right\rangle \quad \text { or } \quad U_{2}=\left\langle x_{\beta}, x_{\alpha+\beta}, x_{2 \alpha+\beta}\right\rangle .
$$

Note that $U_{1}$ is elementary abelian of order 27 . The subgroup $U_{2}$ is an extraspecial group or order 27 and exponent 3. It has elementary abelian subgroups

$$
E_{i}=\left\langle x_{\alpha} x_{\alpha+\beta}^{i}, x_{2 \alpha+\beta}\right\rangle
$$

for $i=0,1,2$ and $E_{3}=\left\langle x_{\alpha+\beta}, x_{2 \alpha+\beta}\right\rangle$. The groups $E_{0}, E_{1}, E_{2}$ are maximal elementary abelian 3-subgroups of $U$, and they are conjugate in $U$ by the powers of the element $x_{\beta}$. Also, $E_{3}$ is not maximal since it is contained in $U_{1}$. Consequently, we have one conjugacy class of maximal elementary abelian 3-subgroup of 3-rank 2 represented by the subgroup $E_{0}$. Notice that, the group $U$ has 3-rank 3. Finally, we should note that $E_{0}$ is not a maximal elementary abelian subgroup in $G$, since it is contained in the elementary abelian 3-subgroup $\left\langle x_{2 \alpha+\beta}, x_{\alpha}, x_{-\beta}\right\rangle$ of $G$.

When $p>3$, every element of $U$ has order $p$. Consider the groups

$$
E_{0} \quad \text { and } \quad F_{r}=\left\langle x_{\alpha} x_{\beta}^{r}, x_{2 \alpha+\beta}\right\rangle \text {, for } 1 \leq r \leq p-1 .
$$

A direct computation shows that the $F_{r}$ 's are self centralizing in $U$ and hence are maximal elementary abelian $p$-subgroups of $p$-rank 2. If $y$ is an element of $U$ that is not in $U_{1}$ or $U_{2}$, then some power of $y$ has the form $y^{t}=x_{\alpha} x_{\beta}^{r} w$ for some $w \in U_{1} \cap U_{2}$ and some $r$. But then $y^{t}$ is $U$-conjugate to $x_{\alpha} x_{\beta}^{r}$. It follows that there are $p$ conjugacy classes of maximal elementary abelian $p$-subgroups of $p$-rank 2 of $U$, represented by the groups $F_{1}, \ldots, F_{p-1}$ and by $E_{0}$.

Next we observe that all of the $F_{r}$ 's are conjugate in $B$, and thus also in $G$. Finally, the subgroup $E_{0}$ is not a maximal elementary abelian $p$-subgroup in $G$, whereas the $F_{r}$ 's remain maximal elementary abelian $p$-subgroups in $G$. Indeed, these are precisely the centralizers of the unique $G$-conjugacy class of the regular elements (all conjugate to $x_{\alpha} x_{\beta}$ ), and thus their centralizers are abelian $p$-groups of $p$-rank equal to the Lie rank of $G$, since $G$ is simple (cf. [Se]).

We summarize our results in the following theorem.

Theorem 8.2. Let $G=B_{2}(p)$. The torsion-free ranks of $T(U), T(B)$ and $T(G)$ are given in the table.

$$
T(U) \quad T(B) \quad T(G)
$$

$\begin{array}{lccc}p=2 & 1 & 1 & 1 \\ p=3 & 2 & 2 & 1 \\ p \geq 5 & p+1 & 3 & 2 .\end{array}$

$\mathbf{G}=\mathbf{G}_{\mathbf{2}}(\mathbf{p})$ : For this group, we adopt the notation in [St], and we use the relations for the generators of the unipotent subgroup $U$ as written out completely on [St, p. 151]. Let $\alpha$ and $\beta$ be the simple roots with $\alpha$ a short root. Set

$$
U_{1}=\left\langle x_{\alpha}, x_{\alpha+\beta}, x_{2 \alpha+\beta}, x_{3 \alpha+\beta}, x_{3 \alpha+2 \beta}\right\rangle \quad \text { and } \quad U_{2}=\left\langle x_{\beta}, x_{\alpha+\beta}, x_{2 \alpha+\beta}, x_{3 \alpha+\beta}, x_{3 \alpha+2 \beta}\right\rangle \text {. }
$$

As in the $B_{2}$-case, we must handle the low primes 2,3 and 5 separately from the larger ones. Let $W=U_{1} \cap U_{2}$. We have the following. 
Lemma 8.3. If $p=2,3$ or 5 , then the unipotent group $U$ has no maximal elementary abelian p-subgroups of p-rank smaller than 3 .

Proof. First notice that if $p=3$, then $x_{2 \alpha+\beta}$ and $x_{3 \alpha+2 \beta}$ are in the center of $U$. Hence maximal elementary abelian 3 -subgroups have 3 -rank at least 3 .

For $p=2$ and $p=5$, we notice that every element of order $p$ in $U$ is either in $U_{1}$ or in $U_{2}$. This follows from the computation of the restricted unipotent variety in [CLNP]. The center of $U_{1}$ is generated by the elements $x_{3 \alpha+\beta}$ and $x_{3 \alpha+2 \beta}$. Therefore, $U_{1}$ has no maximal elementary abelian $p$-subgroup of $p$-rank 2 . The group $U_{2}$ is a central product

$$
U_{2}=\left\langle x_{\beta}, x_{3 \alpha+\beta}\right\rangle *\left\langle x_{\alpha+\beta}, x_{2 \alpha+\beta}\right\rangle
$$

where the factors are dihedral groups when $p=2$ and are extraspecial 5-groups of order 125 and exponent 5 when $p=5$. Consequently, $U_{2}$ is an extraspecial group of order $p^{5}$, and thus it has no maximal elementary abelian $p$-subgroup of $p$-rank 2 .

The lemma now follows from the fact that a maximal elementary abelian $p$ subgroup of $p$-rank 2 in $U$ would have to be contained in either $U_{1}$ or in $U_{2}$.

Assume now that $p \geq 7$. Then, all the nontrivial elements in $U$ have order $p$. As in the previous argument, we have that $U_{1}$ and $U_{2}$ have no maximal elementary abelian $p$-subgroup of $p$-rank less than 3 . So any elementary abelian $p$-subgroup of $p$-rank 2 must be contained in the centralizer of an element of order $p$ that is not in either $U_{1}$ or $U_{2}$. Hence any maximal elementary abelian $p$-subgroup of $p$-rank 2 must be in the centralizer of an element of the form $x_{\alpha} x_{\beta}^{r} w$ for $w \in W$ and $1 \leq r \leq p-1$. Notice that conjugating by an appropriate element of $U$ we can assume that $w=1$. In addition, we know that $x_{\alpha} x_{\beta}^{r}$ is a regular element and so, by the same argument as in the $B_{2}$-case (cf. [Se]), we have that

$$
E_{r}=\left\langle x_{\alpha} x_{\beta}^{r}, x_{3 \alpha+2 \beta}\right\rangle
$$

is a maximal elementary abelian $p$-subgroup of $p$-rank 2 in both $U$ and in $G$. It is easy to see that for all $1 \leq i, j \leq p-1, E_{i}$ is conjugate to $E_{j}$ in $B$ but not in $U$. Therefore, we have proved the following.

Theorem 8.4. Suppose that $G=G_{2}(p)$. Then the torsion-free ranks of $T(U), T(B)$ and $T(G)$ are given in the table.

$$
T(U) \quad T(B) \quad T(G)
$$

$\begin{array}{llll}p=2,3,5 & 1 & 1 & 1 \\ p \geq 7 & p & 2 & 2\end{array}$

\section{REFERENCES}

[A] J. L. Alperin, A construction of endo-permutation modules, J. Group Theory 4 (2001), $3-10$.

[Be1] D.J. Benson, Modular representation theory: New trends and methods, Springer-Verlag Lecture Notes 1081, Springer-Verlag, 1984.

[Be2] D.J. Benson, Representations and cohomology, II, Cambridge Studies in Advanced Mathematics 30, Cambridge University Press, 1991.

[BoCan] W. Bosma, J.Cannon, Handbook on Magma Functions, Sydney University, 1996. 
[BMP] M. Bremner, R. Moody, J. Patera, Tables of dominant weights multiplicities for representations of simple Lie algebras, Marcel Dekker, New York, 1985.

[Ca1] J. Carlson Modules and Group Algebras, Birkhäuser, 1996.

[Ca2] J. Carlson, Constructing Endotrivial Modules, J. Pure and Appl. Algebra, (to appear).

[CLNP] J.F. Carlson, Z. Lin, D.K. Nakano, B.J. Parshall, The restricted nullcone, Cont. Math. 325 (2003), 51-75.

[CaTh1] J. Carlson and J. Thévenaz, Torsion endo-trivial modules, Algebras and Representation Theory, 3 (2000), 303-335.

[CaTh2] J. Carlson and J. Thévenaz, The classification of torsion endo-trivial modules, Annals of Math, (to appear).

[CaTh3] J. Carlson and J. Thévenaz, The classification of endo-trivial modules, Invent. Math., 158 (2), (2004), 389-411.

[C1] R. W. Carter, Simple Groups of Lie Type, John Wiley and Sons, 1972.

[C2] R. W. Carter, Finite Groups of Lie Type: Conjugacy Classes and Complex Characters, John Wiley and Sons, 1985.

[Da] E. C. Dade, Endo-permutation modules over p-groups, I, II, Ann. Math. 107 (1978), 459-494, 108 (1978), 317-346.

[GLS] D. Gorenstein, R. Lyons, R. Solomon, The Classification of the Finite Simple Groups, Volume 40, Number 3, AMS, 1998.

[Hu] B. Huppert, Endliche Gruppen I, Springer-Verlag, 1967.

[Se] G. Seitz, Unipotent elements, tilting modules, and saturation, Inv. Math. 141 (2000), $467-502$.

[St] R. Steinberg, Lectures on Chevalley Groups, (Notes prepared by J. Faulkner and R. Wilson), Yale University, 1967.

Department of Mathematics, University of Georgia, Athens, Ga 30602, USA

E-mail address: jfc@math.uga.edu

Department of Mathematics, University of Georgia, Athens, GA 30602, UsA

E-mail address: nmazza@math.uga.edu

Department of Mathematics, University of Georgia, Athens, GA 30602, USA

E-mail address: nakano@math.uga.edu 